\title{
Neurofilaments as biomarkers in neurological disorders
}

Michael Khalil ${ }^{1 *}$, Charlotte E. Teunissen ${ }^{2}$, Markus Otto $^{3}$, Frederik Piehl ${ }^{4}$, Maria Pia Sormani ${ }^{5}$, Thomas Gattringer ${ }^{1}$, Christian Barro ${ }^{6}$, Ludwig Kappos ${ }^{6}$, Manuel Comabella ${ }^{7}$, Franz Fazekas ${ }^{1}$, Axel Petzold $^{8,9}$, Kaj Blennow ${ }^{10,11}$, Henrik Zetterberg ${ }^{10-13}$, Jens Kuhle ${ }^{6 *}$ [Au: Please write out first names in full for the author list.]

${ }^{1}$ Department of Neurology, Medical University of Graz, Graz, Austria

${ }^{2}$ Neurochemistry Laboratory and Biobank, Department of Clinical Chemistry, Neuroscience

Campus Amsterdam, VU University Medical Center, Amsterdam, The Netherlands

${ }^{3}$ Department of Neurology, Ulm University Hospital, Ulm, Germany

${ }^{4}$ Department of Clinical Neuroscience, Karolinska Institutet, Stockholm, Sweden

${ }^{5}$ Biostatistics Unit, Department of Health Sciences, University of Genoa, Genoa, Italy

${ }^{6}$ Neurologic Clinic and Policlinic, Departments of Medicine, Clinical Research, and Biomedicine, University Hospital Basel, University of Basel, Switzerland

${ }^{7}$ Unit of Clinical Neuroimmunology, Department of Neurology, Hospital Universitari Vall d'Hebron, Universitat Autonoma de Barcelona, Barcelona, Spain.

${ }^{8} \mathrm{UCL}$ Institute of Neurology, Department of Molecular Neurosciences, Moorfields Eye Hospital and The National Hospital for Neurology and Neurosurgery, London, United Kingdom

${ }^{9}$ Departments of Neurology, Ophthalmology and Expertise Center for Neuro-ophthalmology, Amsterdam UMC, Amsterdam, Amsterdam, The Netherlands

${ }^{10}$ Clinical Neurochemistry Laboratory, Sahlgrenska University Hospital, Mölndal, Sweden

${ }^{11}$ Department of Psychiatry and Neurochemistry, Institute of Neuroscience and Physiology, The Sahlgrenska Academy, University of Gothenburg, Mölndal, Sweden

${ }^{12}$ Department of Molecular Neuroscience, UCL Institute of Neurology, Queen Square, London, United Kingdom

${ }^{13}$ UK Dementia Research Institute at UCL, London, United Kingdom

*e-mail: michael.khalil@medunigraz.at,jens.kuhle@usb.ch

\section{Key points:}


- Neuronal damage and loss are the pathological substrate of permanent disability in various acute and chronic neurologic disorders.

- Levels of neurofilament proteins rise upon neuroaxonal damage in cerebrospinal fluid (CSF) and in the blood.

- $\quad 1^{\text {st }}$ generation (immunoblot) and $2^{\text {nd }}$ generation (enzyme-linked immunosorbent assay) neurofilament assays only captured the tip of the ice-berg in disease.

- $3^{\text {rd }}$ generation (electrochemiluminescence) and $4^{\text {th }}$ generation (single molecule array) assays permithighly sensitive longitudinal detection of blood neurofilament levels even in mild disease and from normal controls. [Au: Edited to reduce key point to word limit (30 words). OK?]

- Multicentre studies are underway to consolidate neurofilaments as biomarkers that reflect brain tissue damage, enabling longitudinal monitoring of disease activity and drug effects in clinical trials in neurological diseases. [Au: Edited to reduce key point to word limit (30 words). OK?]

\section{Competing interests}

The authors declare no competing interests related to this article. 


\section{Abstract:}

[Au: Most of my edits to the abstract have been made to reduce the length - the limit is 200 words, and the original was 240 . Please check that you are happy with how it reads and that no crucial information has been removed.]

Neuroaxonal damage is the pathological substrate of permanent disability in various neurological disorders. Reliable quantification and longitudinal follow-up of such damage is important for assessing disease activity, monitoring treatment responses, facilitating treatment development and prognostic purposes. The neurofilament proteins have promise in this context because their levels rise upon neuroaxonal damage not only in the CSF, but also in blood, and they indicate neuroaxonal injury independent of causal pathways. First-generation (immunoblot) and secondgeneration (enzyme-linked immunosorbent assay) neurofilament assays were of limited sensitivity. Third-generation (electrochemiluminescence) and especially fourth-generation (single molecule array) assays enable reliable measurement of neurofilaments throughout the range of concentrations found in blood samples. This technological advancement has paved the way to investigate neurofilaments in a range of neurological disorders. Here, we review what is known about the structure and function of neurofilaments, discuss analytical aspects and knowledge of age-dependent normal ranges of neurofilaments and provide a comprehensive overview of studies on neurofilament light as a marker for axonal injury in different neurological disorders, including multiple sclerosis, neurodegenerative dementia, stroke, traumatic brain injury, amyotrophic lateral sclerosis and Parkinson disease. We also consider work needed to explore the value of this axonal damage marker in managing neurological diseases in daily practice. 


\section{[H1] Introduction}

Neuroaxonal damage and loss are the pathological substrate of many acute and chronic neurological disorders that result in accrual of permanent disability. [Au: Edited to avoid repetition. OK?] The ability to readily detect and follow such damage would be a great advantage in the assessment of disease activity, monitoring of treatment responses and prognosis. [Au: Edited for flow. OK?] Therefore, a biomarker that accurately reflects neuroaxonal injury would be invaluable for reaching individual therapeutic decision and measuring drug effects in clinical trials. Attempts to discover such a biomarker have involved investigation of several avenues, [Au: Beginning of the previous sentence edited to make clear that the avenues relate to the biomarker. OK?] from cerebrospinal fluid (CSF) proteins to MRI, magnetic resonance spectroscopy [Au: Changed to group imaging techniques together. OK?] and metabolic imaging, and have provided different insights with different limitations.

Neurofilaments [Au: We prefer not to abbreviate one-word terms, so I think it's best to not use the abbreviation for neurofilament when it's used alone, but use the abbreviations for $\mathbf{N f L}, \mathbf{N f M}$ and $\mathbf{N f H}$. OK?] are gaining increasing attention as candidate biomarkers of neuroaxonal injury [Au: From here to the end of the paragraph, I have rearranged the information so that it's clear that it's the combination of the specificity and abnormal levels in CSF and serum that makes neurofilaments so attractive as biomarkers. Please check you are happy with how this reads.] because they are abundant structural scaffolding proteins that are exclusively expressed in neurons and that reach pathological levels as a result of axonal damage in neurodegenerative, inflammatory, vascular and traumatic diseases not only in the CSF, but also in serum. The specificity of neurofilaments in terms of cellular source and indication of pathomechanisms means they are highly specific for neuronal cell damage and eventual neuronal cell death, offering a key advantage over other possible biomarkers.

Many, if not all, pathological processes that cause axonal damage release neurofilament proteins into the extracellular fluid, CSF and peripheral blood, depending on the extent of damage. High levels of neurofilaments, [Au: Addition of "high levels of" OK?] therefore, are general indicators of axonal damage irrespective of its cause and any clinical diagnosis, and blood levels of neurofilaments are useful for monitoring and prediction of progression in various acute and chronic neurological diseases and for assessing the efficacy and/or toxicity of treatment. Until recently, measurements of the most promising of the neurofilament proteins, neurofilament light levels in patients with neurological disorders could only be performed with CSF samples, mainly because assay sensitivity was insufficient for reliable quantification of neurofilament light 
levels in the blood. Several studies of CSF [Au: Addition of "of CSF" OK, to relate to the previous sentence more clearly?] have demonstrated that levels of neurofilament proteins are increased in a wide range of neurological diseases ${ }^{1}$. However, given that lumbar puncture is a relatively invasive procedure, longitudinal analyses have been rare and not performed systematically. For the same reasons, neurofilaments have rarely been measured in diseases in which diagnostic lumbar punctures are infrequently indicated. Neurofilament levels in the blood can be quantified with enzyme-linked immunosorbent assay (ELISA) ${ }^{2,3}$ and more-sensitive electrochemiluminescence $(E C L)$ assay technology in many different diseases ${ }^{4,5}$, but neither technique can detect small, disease-related changes. Only the introduction of single molecule array (SiMoA) [Au: This format has been used by other Nature journals for this abbreviation] assays has enabled reliable detection of neurofilament light proteins in blood samples across the whole range of concentrations, including those in healthy individuals ${ }^{6-8}$. Consequently, the past 2 years have witnessed a surge in the number of publications on neurofilament blood levels in a broad range of neurological disorders.

In this Review, we provide background on the structure and function of neurofilaments, consider the analytical aspects of neurofilament measurements and discuss current knowledge on age-dependent normal ranges of neurofilament concentrations. We also review the main neurological disorders in which neurofilament measurements could play a role in research or clinical settings, and highlight aspects that need to be addressed in future studies.

[H1] Neurofilaments - structure and function [Au: Heading shortened to fit our character limits. OK?]

Neurofilaments are classified as intermediate filaments according to their diameter ( $10 \mathrm{~nm})$, which is between that of actin filaments $(6 \mathrm{~nm})$ and myosin filaments (15 nm). Neurofilament heavy chain ( $\mathrm{NfH}, 111 \mathrm{kDa}$ ), [Au: Molecular weights added here to avoid the need for repetition of the subunits later in the paragraph. OK?] neurofilament medium chain ( $N f M, 102.5 \mathrm{kDa}$ ), neurofilament light chain ( $\mathrm{NfL}, 61.5 \mathrm{kDa}$ ) and $\alpha$-internexin $(55.4 \mathrm{kDa})$ belong to the class IV intermediate filaments, and peripherin $(53.7 \mathrm{kDa})$ is a class III intermediate filament (Fig. 1). The molecular weights are higher in vivo owing to an abundance of negatively charged amino acids (glutamic acids) in their sequences and to post-translational modifications ${ }^{11}$. [Au: Statement about molecular weights in vivo moved to here to avoid it being disconnected from the molecular weights after they have been moved to the previous sentence. OK?] 
The neurofilament proteins [Au: Correct, or do you did you mean that class III and IV intermediate filaments contain intrinsically unstructured regions?] contain intrinsically unstructured regions. One key feature of these unstructured regions is that a high proportion of residues are lysine ${ }^{9,10}$; [Au: Additions for clarity. OK?] lysine and serine are the dominant amino acids in the neurofilament tail domain ${ }^{9}$. A relatively conserved, central $\alpha$-helical rod region, a short variable head domain at the amino-terminal end, and a tail of highly variable length at the carboxy-terminal end are highly characteristic for the Nf protein subunits ${ }^{11}$ (Fig. 1). [Au: Subunit list deleted to avoid repetition of what is above. OK?] The head domain contains serine and threonine residues and $\mathrm{O}$-linked glycosylation and phosphorylation sites. The tail domain contains abundant glutamic and lysine-rich stretches of variable length with multiple serine phosphorylation sites. The central rod domain contains hydrophobic repeats that facilitate formation of coil-to-coil dimers.

Formation of neurofilament protein dimers is the first step in heteropolymer assembly. [Au: Sentence added to enable addition of a paragraph break. OK?] Antiparallel aggregation of these dimers leads to formation of tetramers, and eight laterally associated tetramers form the cylindrical unit-length filament (UFL) structure ${ }^{11,12}$. Annealing of UFLs leads to longitudinal elongation of neurofilaments, which is followed by radial compaction to form the final long neurofilaments with diameters of $10 \mathrm{~nm}$ (Fig. 1$)^{12}$.

Post-translational modifications of neurofilaments include addition of $\mathrm{O}$-linked $\mathrm{N}$ acetylglucosamine (O-GIcNAc) to individual serine and threonine residues, nitration, oxidation, ubiquitination and most importantly phosphorylation ${ }^{11,13}$. All subunits are phosphorylated on their head domain, but only NfM and NfH are extensively phosphorylated on their carboxyterminal domains, and this phosphorylation increases the resistance of these subunits to proteases ${ }^{14}$. [Au: Does this edit retain your meaning? Or do you mean that the fact these subunits are phosphorylated makes them more resistant to proteases than the other subunits?] Under normal conditions, neurofilaments are highly stable within axons, and their turnover is low. The filaments form a liquid crystal gel network with in diseases like ALS, Lewy-body-based dementia or Parkinson's disease, neurofilament accumulation [Au: Please clarify what you mean by compartmental accumulation - where is this accumulation, and what compartments are being referred to?] related to subunit stoichiometry and the degree of phosphorylation ${ }^{14}$.

The precise functions of neurofilaments remain unknown, but they are thought to be critical for radial growth and stability of axons, thereby enabling effective, high-velocity nerve conduction ${ }^{15,16}$. Several reports indicate that neurofilaments interact with other proteins and 
organelles, including mitochondria and microtubules ${ }^{11}$, suggesting that they have important functions beyond preserving axonal stability.

Several mutations identified in the genes that encode neurofilament proteins can lead to abnormal neurofilament aggregation and accumulation with the consequence of axonal dysfunction and neurodegeneration. For example, mutations in the NEFL gene, which encodes NfL, lead to Charcot-Marie-Tooth Neuropathy Type 2E/1F (CMT2E/1F) [Au: Please make clear what the two abbreviations mean specifically] disease. Mutations of the genes that encode peripherin $(P R P H), \mathrm{NfH}(N E F H)$ and NfM (NEFM) have been associated with increased susceptibility to amyotrophic lateral sclerosis (ALS) and familial Parkinson disease (PD). Mutations in genes other than those that encode neurofilament proteins can have secondary effects on neurofilament aggregation; such mutations include those in heat-shock 27-kDa protein 1 in CMT2F, gigaxonin in giant axonal neuropathy and superoxide dismutase 1 (SOD1) in ALS ${ }^{11,17}$.

\section{[H1] Assays to detect soluble neurofilaments}

In the past three decades, impressive advances have been made in the development of sensitive immunoassay technologies. With these advances, detection of neurofilaments has improved (Fig. 2), [Au: See my suggestion for making Figure 1c a separate Figure 2] moving towards evermore clinically useful capabilities. [Au: I have added the previous sentence firstly because I felt it was helpful to relate the advances to neurofilament in a general sense at the outset, and also because I think it would be nice to have each generation discussed in its own paragraph, but breaking the paragraph here left one sentence in the first paragraph, which is not ideal. Addition OK?]

First-generation [Au: Unfortunately, our style does not allow use of italics for emphasis] immunoassays were semi-quantitative at best. Immunoblots based on electrophoretic protein separation or dot blots were, however, consistent in that they reliably demonstrated the presence of neurofilament isoforms in the CSF and blood of patients with a range of diseases ${ }^{10}$.

Second-generation sandwich ELISA technology produced the first reliable quantitative data that enabled assessment of the prognostic and diagnostic value of $\mathrm{NfH}$ and $\mathrm{NfL}$ in the CSF in human disease $\mathrm{e}^{2,18-20}$. Human body fluid compartments that were analysed with this technique extended to the interstitial and extracellular fluid ${ }^{21}$, serum and plasma, amniotic fluid and the vitreous body ${ }^{22}$. Meta-analyses and international validation studies demonstrated that high precision could be achieved in expert laboratories, but also highlighted the need for improved assay standardization ${ }^{23,24}$. [Au: Standardization of what? Please clarify] 
Third-generation ECL technology led to a substantial improvement in the analytical sensitivity 4, 5, 25-27. ECL based assays are known to be highly sensitive, exhibit a broad dynamic range and require small sample volume, however we found the SiMoA technology to be 126- and 25-fold more sensitive than ELISA and the ECL assay, respectively, to quantify $\mathrm{NfL}^{7}$. [Au: Is it possible to expand on this statement to explain more about the technology, why it improved sensitivity and the limitations it still had?]

Finally, fourth-generation SiMoA technology improved analytical sensitivity to an extent that reliable quantification of NfL levels in blood became possible across the range of concentrations that are observed in disease and in physiological conditions ${ }^{6-8,28}$. This cutting-edge method is based on single-molecule arrays and the simultaneous counting of singulated capture microscopic beads (2.7 $\mu \mathrm{m}$ diameter) carrying sandwich antibody complexes. [Au: Please clarify for non-experts what these microbeads are - presumably neurofilament proteins bind to them?] The analytical sensitivity is manifold higher than with use of the same antibodies in the ELISA format designed for CSF measurements ${ }^{19}$, and enables reliable measurement of the low $\mathrm{NfL}$ concentrations in blood samples from young healthy individuals ${ }^{6,8}$ so that minor changes in levels of this protein that occur in normal ageing or after mild injury [Au: Change from "concussion" to mild injury OK?] can be monitored. Close correlation between NfL levels in the serum or plasma with levels in the CSF, which has been demonstrated in numerous studies and various neurological diseases, allows conclusions about the degree of ongoing neuroaxonal injury to be drawn from blood levels without the need to obtain CSF by lumbar puncture ${ }^{4,8,29-36}$. Investigations of NfM have been sparse ${ }^{37}$, but commercial SiMoA kits for detection of $\mathrm{NfL}$ and phosphorylated $\mathrm{NfH}$ are available.

[H1] Neurofilaments in ageing [Au: Edited to fit our character limits for headings and also because only ageing is discussed in this section. OK?] Normal ageing is associated with neurodegenerative processes that can be detected with various markers such as volumetric loss of brain tissue [Au: Here, I think the wording raises the question for the reader of why neurofilaments are needed as a marker if neurodegeneration can be detected with other markers. I assume the existing markers, such as imaging, are more difficult and more costly and the neurofilament could be detected with a simple blood test - correct? If so, I think it would be helpful to explain this so that the reader is clear about the need for neurofilament.] but also by increased levels of a range of fluid biomarkers comprising neurofilaments. The advantage of an easy to access body fluid biomarker, such as neurofilament in 
the blood is to provide a real-time signal on neuro-axonal damage of the entire CNS, paralleled by lower costs and the ability of repeated measurements in a relatively non-invasive manner (Barro et al. Brain 2018). In CSF, the normal upper reference value for NfL levels increases 2.5-fold between the ages of 20 years and 50 years, and doubles further by the age of 70 years ${ }^{38}$. This agerelated increase in levels in the lumbar CSF could be due to reduced CSF turnover ${ }^{39}$, as a general physiological phenomenon ${ }^{39}$ [Au: Did you mean that reduced CSF turnover is a general physiological phenomenon? I have removed on the basis of this, as I felt it was not necessary, but if you meant a general physiological phenomenon to be a separate item in the list, please clarify] but could also indicate slow, ongoing axonal injury. The latter possibility is supported by the finding that CSF levels of $\mathrm{NfL}$ in cognitively healthy elderly individuals correlate with hippocampal atrophy independently of age and AD biomarkers ${ }^{40}$. However, a detailed understanding of the mechanisms that underlie age-related increases in neurofilament levels is lacking. Besides structural damage and loss of neurons, metabolic alterations in the turnover of neurofilament proteins might play a role: experimental evidence demonstrates complex changes in the expression of mRNA, post-translational mRNA modification [Au: "post translation" removed from here because it did not seem to relate to anything. If it is necessary, please clarify what it meant.] and neurofilament protein turnover ${ }^{41}$.

A highly significant correlation is also seen between age and NfL blood levels: use of [Au: "fourth generation" removed from here because it implied that there have been four generations of SiMoA technology.] SiMoA technology has shown that $\mathrm{NfL}$ levels in the blood increase by $2.2 \%$ per year between the ages of 18 years and 70 years ${ }^{8,42}$. The strong correlation between CSF and blood levels of $\mathrm{NfL}$ at the group level suggests that the two measures reflect similar physiological processes ${ }^{4,8,29-36}$. [Au: Change from "factors" to "physiological processes" OK?] Nevertheless, important to acknowledge is the possibility that degenerative processes in the PNS contribute to neurofilament levels in peripheral blood ${ }^{4,43,44}$.

Important for further development of neurofilaments as a biomarker is to establish universal reference values for healthy controls by analysis of samples under standardized and controlled conditions in reference laboratories. These reference values would enable correct interpretation of levels seen in various pathological conditions, thereby maximizing the potential of neurofilaments in the management of [Au: Addition of "the management of" OK?] diseases that involve neuroaxonal injury. 
[H1] Neurofilaments in neurological disease [Au: Edited to fit our character limits for headings. OK?]

CSF and blood levels of neurofilament proteins have been measured in various neurological disease (Box 1), and evidence has accumulated that they can be clinically useful biomarkers in many of these. Below, we discuss the evidence in each of the studied diseases. [Au: I have added this paragraph to provide a short introduction to this main section and also to provide an opportunity to cite Box 1. Please see my suggestion for converting Figure 2 to Box 1 below.]

\section{[H2] Multiple sclerosis}

Multiple sclerosis (MS) is a chronic disease of presumed autoimmune origin that is, at least initially, characterized by episodes of focal inflammation in the brain and spinal cord that predominantly involve the white matter but can involve the grey matter ${ }^{45}$. Formation of new lesions can be visualized with MRI, the only established biomarker of disease activity used in routine clinical practice today. However, MRI primarily detects lesions in the white matter, and grey matter damage is largely missed with standard imaging techniques ${ }^{46,47}$. In addition, MRI does not allow selective detection of neuroaxonal degeneration, which seems to be the most important determinant of long term disability ${ }^{48-50}$. Several MRI-based volumetric measures, including analysis of cortical thickness, have been used to assess neuronal degeneration, but the specificity and sensitivity of these measures at the individual level are limited ${ }^{51}$

Use of second-generation immunoassays to measure NfL, pioneered by Rosengren et al. ${ }^{18,52}$, revealed three key aspects of disease associated with CSF levels of NfL: [Au: Edited for clarity about what the relationships are between. OK?] the degree of disability, disease activity, and the time since the last relapse in patients with relapsing-remitting MS (RRMS) ${ }^{52}$. These initial findings were replicated and extended by subsequent, larger studies of clinical aspects associated with CSF levels of $\mathrm{NfL}^{53-56}$ and $\mathrm{NfH}^{26,54,57,58}$.

Use of third-generation immunoassays further revealed that CSF levels of $\mathrm{NfL}$ reduce as a consequence of disease-modifying therapy (DMT). For example, initiation of the high-efficacy DMT natalizumab resulted in normalization of CSF NfL levels to those seen in healthy controls within 612 months ${ }^{59}$, suggesting that NfL can be used to monitor therapeutic efficacy. Similar observations were made in placebo-controlled ${ }^{60}$ and observational ${ }^{61,62}$ studies of fingolimod in patients with RRMS and in studies of mitoxantrone and rituximab ${ }^{63}$ and of natalizumab ${ }^{64}$ in progressive MS. 
Despite the promising results in MS, a major barrier to widespread adoption of $\mathrm{NfL}$ analysis in MS research and clinical practice has been the requirement of CSF sampling, but this problem has finally been overcome by use of fourth-generation immunoassays. Of particular interest is the demonstration that serum levels of $\mathrm{NfL}$ can be used to separate not only patients with MS from healthy controls, but also patients with MS who have enhancing MRI lesions from patients without such lesions ${ }^{8}$. Furthermore, serum NfL levels in patients with MS have been independently associated with disability and relapse status ${ }^{8,}$ Barro et al., Brain 2018, [Au: Please cite the appropriate reference(s) to support this statement] and the risk of future relapses and disability worsening is higher among patients with high serum levels of $\mathrm{NfL}$ than those with lower levels ${ }^{8}$ Barro et al Brain 2018 . [Au: Please cite the appropriate reference(s) to support this statement] Finally, patients with ongoing DMT had lower serum NfL concentrations than did untreated patients ${ }^{8}$. Yet another study found that patients who switched from injectable therapies to [Au: Edited because it wasn't clear what injectable therapies are less effective in comparison to until you continued, whereas this wording makes clear that fingolimod is more effective than injectables. OK?] fingolimod had significantly lower serum NfL levels than when they were on injectables [Au: Lower than levels when they were on injectables, or lower than patients who continued on injectables? Please clarify] over a 2-year period ${ }^{30}$. Associations with disease activity and treatment-related reductions in serum NfL levels were confirmed by another observational study in which a fourth-generation immunoassay was used in a large, independent cohort of patients with RRMS ${ }^{36}$. Recently, a longitucinal observational study demonstrated that patients with increased serum $\mathrm{NfL}$ at baseline, independently of other clinical and MRI variables, experience significantly more brain and spinal cord volume loss over 2 and 5 years of follow-up (Barro et al., Brain, 2018).

Collectively, the findings described (Table 1) make a strong case for bringing fourthgeneration serum $\mathrm{NfL}$ assays from the bench to the clinics in the management of MS. Further studies are required to show how these assays can be used for monitoring disease activity and for therapeutic decision-making.

\section{[H2] Dementias}

Dementia - defined in this context as cognitive disturbances that are severe enough to interfere with activities of daily living - can be caused by several different neurodegenerative disorders, of which Alzheimer disease (AD) is the most prevalent, frontotemporal dementia (FTD) is the secondmost prevalent among people aged $<60$ years, and dementia with Lewy bodies (DLB) is the second-most prevalent among patients aged >60 years. [Au: Edited to define "older" on the basis 
of the definition of "younger" in the previous sentence. OK?] Currently, clinical diagnosis of the different types of dementia relies largely on documenting cognitive decline or on post-mortem evaluation. However, it is becoming clear that early brain damage occurs decades before the onset of clinical symptoms. This observation opens a window of opportunity for secondary prevention and suggests the value of [Au: Change of wording OK?] a shift from using clinical hallmarks for diagnosis to monitoring of biological measures that reflect ongoing pathological processes. Several studies have addressed the question of whether neurofilaments can provide such a biological measure (Table 2). [Au: Sentence added to enable citation of Table 2. OK?]

An early study in 1999 demonstrated a mild increase in CSF levels of NfL in AD, and substantially higher levels in FTD ${ }^{66}$. These findings were confirmed in a subsequent study that also showed that the increase in CSF levels of NfL in AD is seen only in patients with late-onset disease, [Au: Edit to avoid referring to patients as "cases"] whereas NfL levels are not significantly different from normal controls [Au: Edited to specify controls - correct?] in patients with early onset $A D^{67}$.

Subsequent meta-analyses of findings obtained with second-generation immunoassays consistently demonstrated that CSF levels of $\mathrm{NfL}$ are increased in the mild cognitive impairment and dementia stages of $A D^{23,68}$ and are independent of $A \beta$ load ${ }^{68,69}$. [Au: Edited for clarity. OK?] The diagnostic specificity of NfL levels was lower than the hallmark $A D$ biomarkers of $A \beta_{1-42}$ levels, [Au: OK?] $A \beta_{1-42}: A \beta_{1-40}$ ratio and phosphorylated tau levels ${ }^{68,70}$. Nevertheless, evidence indicates that NfL levels correlate with and are predictive of brain atrophy and worsening of cognition independently from $A \beta$ pathology ${ }^{69,71}$. Moreover, $\mathrm{NfL}$ levels in the blood have some predictive value for progression to $A D$ dementia in patients with subjective memory complaints ${ }^{42}$, so the potential for use of $\mathrm{NfL}$ levels in combination with clinical evaluation and other biomarkers to detect the earliest stage of the disease should be assessed. Furthermore, the greatest value of NfL in $A D$ dementia could be in monitoring responses to treatment, as in $M S$, as reductions in plasma $\mathrm{NfL}$ [Au: Please explain what was observed - presumably NfL levels decreased?] were observed in animal models of AD when treated with a BACE-inhibitor ${ }^{31}$.

Measurement of NfL levels is also likely to be of value in the diagnosis of FTD, in which CSF $^{23,67}$ and serum ${ }^{35,75}$ levels of NfL [Au: CSF levels of NfL correct?] are high and approach those observed in ALS (see Amyotrophic lateral sclerosis section below). Indeed, among the chronic dementias, the highest CSF NfL concentrations are observed in FTD and vascular dementia, followed by $A D^{23,72}$. Among patients with FTD, CSF levels of NfL are higher in those with TAR DNAbinding protein 43 (TDP-43) inclusions than in those with tau pathology (confirmed by genetic 
testing or post-mortem evaluation $)^{73}$. Moreover, CSF levels of $\mathrm{NfL}$ increased when symptoms developed in patients with genetic FTD, and these levels were inversely correlated with survival ${ }^{35}$. Several studies have confirmed a strong relationship between CSF and serum levels of $\mathrm{NfL}$ and the time to death in patients with FTD 35,73 .

The results of fourth-generation immunoassays ${ }^{74}$ for detection of neurofilaments reflect neuroaxonal damage in neurodegenerative dementias, including $\mathrm{FTD}^{75}$, familial and sporadic $A D^{42}$, 76 and atypical parkinsonian disorders ${ }^{77}$. In sporadic AD, plasma $\mathrm{NfL}$ concentrations are already increased in the mild cognitive impairment stage, and correlate with cognitive, biochemical and imaging hallmarks of the disease ${ }^{42}$. In familial $A D$, blood $\mathrm{NfL}$ concentrations start to increase $\sim 10$ years before the expected onset ${ }^{76}$.

Very high CSF and blood levels of NfL [Au: This is a little confusing, because CJD has not been mentioned earlier in the section, and earlier in the section, it's stated that the highest levels of NfL are seen in FTD among the dementias. This conflict either needs to be resolved, or the discussion of CJD needs to be omitted. Also, does "highest" here mean within dementias, or the highest in any condition?] have been observed in patients with sporadic and familial Creutzfeldt-Jakob disease. In this condition, CSF levels of NfL were increased before symptom onset, and the sensitivity and specificity of serum $\mathrm{NfL}$ concentration for diagnosis of Creutzfeldt Jakob disease [Au: Addition correct, to ensure it's clear what the sensitivity and specificity relate to?] were $100 \%$ and $85.5 \%$, respectively ${ }^{78}$. Elevated serum levels of $\mathrm{NfL}$ have also been described in patients with primary progressive aphasia ${ }^{79}$; [Au: Please cite the appriopriate reference(s) to support the previous statement] higher levels were identified in patients with the non-fluent or agrammatic and semantic variants than in those with the logopenic variant. $\mathrm{NfL}$ levels correlated with clinical progression and brain volume loss in all patients with primary progressive aphasias ${ }^{79}$. [Au: Does the last sentence relate to all three variants, or only the non-fluent and semantic variants? Please clarify.]

\section{[H2] Stroke}

Most existing data on neurofilaments in stroke are CSF measurements in subarachnoid haemorrhage (SAH). Studies have shown that $\mathrm{NfH}$ and $\mathrm{NfL}$ levels are higher among patients with aneurysmal SAH than among healthy controls or patients free of neurological disease ${ }^{80-82}$. [Au: Please define the controls more specifically - were they healthy?] The exact causes of neurofilament elevation in SAH in the absence of associated focal lesions (parenchymal haematoma or ischaemia owing to vasospasm) are not entirely clear, but are presumably 
attributable to diffuse neuroaxonal injury or iatrogenic following for eaxample placement of an external ventricular drain. [Au: Please clarify what you mean by "neurosurgical procedures"] Regardless, evidence suggests that neurofilament levels consistently correlate with the clinical severity and extent of morphological brain damage ${ }^{80,81}$.

The ability to analyse neurofilament light levels in blood samples with fourth-generation immunoassays has facilitated the study of this marker in stroke in which a lumbar puncture is usually not indicated. [Au: Please clarify. Do you mean that lumbar puncture is not indicated in some subtypes, or do you mean that lumbar puncture is not indicated in stroke generally? And by "not indicated", do you mean it is contra-indicated, or there is just no reason to do it?] This approach has been used to show that serum levels of $\mathrm{NfL}$ are higher among patients with spontaneous cervical artery dissection who had an ischaemic stroke than among those with transient ischaemic attacks or isolated local symptoms ${ }^{83}$. [Au: Change to "isolated" OK?] Similarly, serum $\mathrm{NfL}$ concentration was found to be increased in patients with a single, recent, small subcortical infarct compared with concentration in age-matched and sex-matched healthy controls $^{84}$. In the same study, assessment of the temporal dynamics of $\mathrm{NfL}$ at 3 months and 15 months after stroke revealed especially high levels in patients with new, clinically silent brain lesions related to small vessel disease detected with MRI during follow-up, suggesting that NfL levels indicate active small vessel disease. Interestingly, serum NfL levels increased during the first few days after stroke onset and remained elevated in a follow-up assessment at 3 months. Comparable findings of neurofilament dynamics have been reported in other studies ${ }^{5,83,85}$. Prolonged release of NfL into the blood after acute neuronal injury might be caused by persistent blood-brain barrier breakdown, but ongoing post-ischaemic immunological or inflammatory processes could also explain these findings.

\section{[H2] Traumatic brain injury}

Mild traumatic brain injury (TBI), also called concussion, is caused by non-penetrating head trauma and is increasingly recognized as a major health problem ${ }^{86}$. Most patients with mild TBI recover within hours to days, but a percentage have symptoms for weeks to months after the head impact, a condition called post-concussive syndrome. Furthermore, an unknown proportion of people who are exposed to repeated concussions, primarily contact sports athletes such as boxers and American football players and soldiers who are exposed to explosive blasts, develop a chronic neurodegenerative disease called chronic traumatic encephalopathy $(\mathrm{CTE})^{87}$, previously known as dementia pugilistica ${ }^{88}$. 
Mild TBI and post-concussive syndrome are vaguely defined clinical entities and their diagnosis is based only on the presence of one or several unspecific symptoms (such as loss of consciousness, dizziness, headache and poor concentration), causing a major issue in research, clinical management and drug development in this field ${ }^{86}$. Consequently, sensitive biomarkers are needed to identify and grade neuronal injury in individuals with mild TBI and post-concussive syndrome. Furthermore, biomarkers that enable grading of severity of neuronal injury after a mild TBI might be important as objective tools for guiding sports physicians with return-to-play decisions for their athletes.

Studies of contact sports athletes with mild TBI show that CSF levels of NfL increase more than levels of tau, suggesting that minor head injuries affect long myelinated white-matter axons more than they affect shorter cortical axons ${ }^{89,90}$. In severe TBI, fourth-generation NfL immunoassays ${ }^{6}$ have demonstrated a marked increase in blood NfL levels that also predicted clinical outcome ${ }^{91}$, thereby confirming earlier findings from third-generation immunoassay studies of CSF and blood samples ${ }^{92}$. Interestingly, marked increases in blood NfL levels have been detected in amateur boxers after a bout; higher $\mathrm{NfL}$ levels were seen in boxers who had received more head impacts, and levels approached normalization after 3 months of rest from boxing ${ }^{93}$. Similarly, blood levels of $\mathrm{NfL}$ were found to increase during the course of a season in American football players ${ }^{94}$. Taken together, these results (Table 3 ) support the idea that the blood level of $\mathrm{NfL}$ is a sensitive indicator of axonal injury after mild TBI and is a promising candidate for clinical application and contact sports medicine.

\section{[H2] Amyotrophic lateral sclerosis}

Motor neuron diseases are neurodegenerative disorders characterized by degeneration of the upper and lower motor neurons, and the most common form is $\mathrm{ALS}^{95}$. Given that axonal impairment can be seen early in the disease, measurement of neurofilaments in the CSF of patients with ALS was an obvious and straightforward experiment and led to the observation that $\mathrm{NfL}$ levels are increased in this condition ${ }^{18,96,97}$.

Several independent studies have confirmed that neurofilament levels are significantly elevated in patients with ALS compared with several other disorders (Table 4); the largest prospective study included 455 patients ${ }^{34,98-103}$. Diagnostic sensitivities and specificities were up to $\sim 80 \%$. Higher levels were also associated with faster disease progression. Increases in $\mathrm{NfL}$ and $\mathrm{NfH}$ levels were also observed in the early clinical phase of patients with genetic ALS and in patients with sporadic ALS ${ }^{33}, 104-107$. The first clinical sign of the disease seems to be associated with a 
massive increase of neurofilament levels in the $\mathrm{CSF}^{33}$, and a corresponding increase in NfL levels has been observed in the blood ${ }^{33}$. Furthermore, increased levels of blood $\mathrm{NfH}$ have been seen in patients with sporadic ALS 100, 102, 108 . The difference in dynamics of higher NfL levels if compared to $\mathrm{NfH}$ levels in ALS and controls may partly be explained by earlier assay sensitivity issues. A new hypothesis, adaptive protein stoichiometry, suggests that the neurodegenerative process itself alters the quantitative relationship of neurofilament subunits. This leads to a relative over expression of $\mathrm{NfL}$ compared to $\mathrm{NfM}$ and $\mathrm{NfH}$ in order to minimise ATP requirements for subunit translation in the motorneuron [Zucchi et al. Journal Neurochemistry, 2018]. [Au: Edited for clarity. OK?]

Although helpful for diagnostic purposes, the reason for the very high CSF levels of neurofilaments in ALS is still not entirely clear, even under the assumption that neurofilament levels reflect neuroaxonal damage. One small study showed a correlation of $\mathrm{NfL}$ levels with axonal impairment assessed with diffusion tensor imaging (DTI) ${ }^{109}$, [Au: Edited to streamline. OK?] but this correlation was not seen in a similar study that included 75 patients ${ }^{101}$. [Au: Edited to clarify and simplify. Is there a significance to the number of patients in the second study - is it much larger than the number in the first study? Please make this clear, because I think it is important to indicate to the reader which of these two studies is likely to be more reliable.] NfL concentrations in the blood seem to be stable at a very high level during follow-up of patients with ALS, whereas DTI values increase ${ }^{34,79}$; only [Au: Addition of "only" OK?] a slight increase in blood levels of $\mathrm{NfH}$ has been described ${ }^{99}$. One mechanistic explanation is based on evidence that TDP-43, the major neuropathological hallmark of ALS, directly interacts with neurofilament production and causes [Au: This wording suggests that the protein causes the release. Do you mean the aggregation of TDP-43 causes the release, or another aspect of TDP-43 function/pathology?] the sudden and massive release of neurofilaments in $\mathrm{ALS}^{110}$. More prospective studies of neurofilament levels in ALS, especially in the blood, are needed. [Au: Please expand on this sentence to say why they are needed - to determine the mechanisms, or for translation of the findings to clinical use?]

\section{[H2] Parkinson disease}

Although Parkinson disease (PD) is one of the most common neurodegenerative disorders, no validated neurochemical biomarkers are currently available to aid clinical diagnosis. In PD and other synucleinopathies, $\alpha$-synuclein is the main component of [Au: OK?] neuronal inclusions. Many studies have been performed to assess whether $\alpha$-synuclein in the CSF could be an effective 
biomarker of PD; ELISA has been used in most of these studies, which have produced contradictory results ${ }^{112,113}$. [Au: Sentence edited for clarity. OK?]

In 1998, NfL was first investigated in the CSF of 49 patients [Au: Number of patients added here. OK?] undergoing differential diagnosis for a Parkinsonian syndrome, including patients with atypical parkinsonian syndromes such as progressive supranuclear palsy (PSP) and multiple system atrophy (MSA). These investigations demonstrated increased CSF NfL levels in PSP and MSA compared with the PD group patients ${ }^{114}$. [Au: Correct that the levels were increased in all of these patients?] This increase in PSP and MSA versus PD was also seen for $\mathrm{NfH}^{115}$. In a larger study that included $>450$ patients with PSP, MSA or PD, almost no overlap was seen between CSF levels of $\mathrm{NfL}$ in patients with atypical parkinsonian disease and those with PD; NfL levels were increased mainly in the atypical disorders ${ }^{116}$. The finding was validated in an independent cohort ${ }^{117}$.

In a study published in 2016, high levels of NfL were observed in blood of patients with PSP, and this difference persisted at one year follow-up. [Au: Correct that the follow-up was after treatment? How long was the follow-up period? How were they treated? Please add more detail so that findings are clearer] Patients with higher NfL levels had more severe neurological, functional, and neuropsychological deterioration over 1 year. Higher baseline NfL predicted greater whole-brain and superior cerebellar peduncle volume loss ${ }^{118}$. On the basis of these findings, the investigators concluded that NfL could be used not only to aid diagnosis, but also to monitor pharmacodynamic effects, especially in clinical trials. The findings of this study were also validated in three independent cohorts, leading to the suggestion that $\mathrm{NfL}$ could be used in both primary care and specialized clinics ${ }^{77}$.

[H2] Huntington disease [Au: I didn't see any reason to group HD and bipolar disorder together.] Huntington disease (HD) is a progressive neurodegenerative disorder caused by CAG repeat expansions in the HTT gene, leading to the formation of mutant huntingtin (mHTT). No proven disease-modifying treatments yet exist ${ }^{119}$. The slow and insidious progression of neurodegeneration in HD has made it challenging to detect disease-related changes in the levels of neurofilament proteins in the blood ${ }^{120}$. However, increased CSF levels of NfL have been demonstrated in patients with $\mathrm{HD}^{121,122}$, and fourth-generation technology has revealed a strong relationship between plasma levels of NfL, HD onset and subsequent progression of neurodegeneration ${ }^{119}$. If confirmed, blood $\mathrm{NfL}$ levels could be included as a secondary outcome measure in future clinical trials in HD. 
[H2] Bipolar disorder [Au: We don't generally cover psychiatric conditions, but I do feel it is interesting to include this because the fact that there are some changes in a disease associated with some degeneration reinforces the association of the marker with neuronal injury. However, I have suggested some changes to wording to emphasize the aspects that will probably be of most interest to our audience. Please check that you are happy with these changes.]

Some evidence suggests that neurodegeneration and neuroaxonal injury can be associated with some subtypes of bipolar disorder ${ }^{123}$. Although these aspects are not prominent features of the condition, CSF levels of NfL were slightly increased in a subset of patients, particularly those who are treated with atypical antipsychotics ${ }^{124}$, presumably reflecting a not yet fully understood disease-associated or treatment-associated effect. [Au: Addition made to emphasize the focus on the biological/mechanistic aspects. OK?] However, no clear relationship was seen between NfL levels and clinical outcomes, such as manic or hypomanic and depressive episodes (cross-sectional data), suicide attempts, psychotic symptoms or inpatient care ${ }^{125}$. Although the current evidence for detection of neuroaxonal injury in bipolar disorder by measuring neurofilaments is limited, the available results warrant longitudinal studies of well-characterized patients to examine how neurofilament concentrations change over time in relation to disease activity and phase (depression and mania) and whether neurofilaments can indicate adverse effects of treatments. Fourth-generation measurement technology will facilitate such studies by enabling measurements to be taken from blood samples.

[H1] Conclusions and future aspects [Au: The last section of all our Reviews must contain the word "conclusions". Change of heading OK?]

In summary, highly sensitive neurofilament measurements have the potential to fill a gap in the assessment of neuroaxonal damage in various neurological disorders. For the first time, this approach provides a sensitive assessment of the consequences of brain tissue damage with only a blood sample, an important advance to aid research and towards use of the assays in clinical practice. [Au: Edited to give the importance of the advance a little more weight?] In relation to clinical trials, the reviewed characteristics of neurofilaments, especially of $\mathrm{NfL}$, make these proteins optimal candidates as markers of outcome in phase II trials in neurological disorders. Definitive phase III trials must use clinical endpoints (clinical events with a clear effect on the duration or quality of life) to confirm a clinical benefit, but the aim of phase II trials is to identify 
drugs with sufficient activity to continue to phase III, so earlier end points are preferable. [Au: Information about trial end points edited to improve flow. OK?] [Au: The previous sentence has been removed, as this passage felt a bit repetitive. OK?]

To validate neurofilament measurements as phase II trial end points, two additional properties must be verified: a correlation with the clinical end points used in phase III trials, and an ability to detect a treatment effect. To verify these properties, a promising approach is retrospective analysis of data from randomized clinical trials in which blood samples suitable for measurement of neurofilaments have been collected. Comparison of neurofilament levels between subgroups of patients enrolled in the trials would determine whether the drug tested had an effect on the neurofilament biomarker. Moreover, neurofilament levels and their stoichiometry could be correlated with all the other relevant clinical and para-clinical measures collected in the trial.

The main factors limiting application of neurofilament measurements to disease monitoring individuals are the lack of normal values of neurofilament across all age groups, a detailed understanding of how comorbidities affect blood neurofilament measurements, and the need for thorough multicentre analytical assay validation to achieve standardized and reliable measurements across different sites. In light of the effect of ageing on neurofilament levels, generation of normative data in large collections of controls is a priority. Co-ordinated multicentre research activities are already ongoing to tackle these obstacles.

[Au: For references that are particularly worth reading (5-10\% of the total), please provide a single bold sentence that indicates the significance of the work.]

1. Deisenhammer, F. et al. EFNS guidelines on disease-specific CSF investigations. Eur. J. Neurol 16, 760-770 (2009).

2. Petzold, A., Keir, G., Green, A.J., Giovannoni, G. \& Thompson, E.J. A specific ELISA for measuring neurofilament heavy chain phosphoforms. J. Immunol. Methods 278, 179-190 (2003).

3. Petzold, A., Rejdak, K. \& Plant, G.T. Axonal degeneration and inflammation in acute optic neuritis. J. Neurol. Neurosurg. Psychiatry 75, 1178-1180 (2004).

4. Gaiottino, J. et al. Increased neurofilament light chain blood levels in neurodegenerative neurological diseases. PLoS. One 8, e75091 (2013).

5. Kuhle, J. et al. Serum neurofilament light chain is a biomarker of human spinal cord injury severity and outcome. J. Neurol. Neurosurg. Psychiatry 86, 273-279 (2015). 
6. Gisslen, M. et al. Plasma Concentration of the Neurofilament Light Protein (NFL) is a Biomarker of CNS Injury in HIV Infection: A Cross-Sectional Study. EBioMedicine 3, 135-140 (2016).

7. Kuhle, J. et al. Comparison of three analytical platforms for quantification of the neurofilament light chain in blood samples: ELISA, electrochemiluminescence immunoassay and Simoa. Clin Chem Lab Med 54, 1655-61 (2016).

8. Disanto, G. et al. Serum Neurofilament light: A biomarker of neuronal damage in multiple sclerosis. Ann Neurol 81, 857-870 (2017).

9. Dyson, H.J. \& Wright, P.E. Intrinsically unstructured proteins and their functions. Nat. Rev. Mol. Cell Biol 6, 197-208 (2005).

10. Petzold, A. Neurofilament phosphoforms: surrogate markers for axonal injury, degeneration and loss. J. Neurol. Sci 233, 183-198 (2005).

11. Yuan, A., Rao, M.V., Veeranna \& Nixon, R.A. Neurofilaments and Neurofilament Proteins in Health and Disease. Cold Spring Harb. Perspect. Biol 9 (2017).

12. Herrmann, H. \& Aebi, U. Intermediate Filaments: Structure and Assembly. Cold Spring Harb. Perspect. Biol 8 (2016).

13. Nixon, R.A. \& Sihag, R.K. Neurofilament phosphorylation: a new look at regulation and function. Trends Neurosci 14, 501-506 (1991).

14. Beck, R., Deek, J. \& Safinya, C.R. Structures and interactions in 'bottlebrush' neurofilaments: the role of charged disordered proteins in forming hydrogel networks. Biochem. Soc. Trans 40, 1027-1031 (2012).

15. Barry, D.M. et al. Expansion of neurofilament medium $C$ terminus increases axonal diameter independent of increases in conduction velocity or myelin thickness. J. Neurosci 32, 6209-6219 (2012).

16. Rao, M.V. et al. The neurofilament middle molecular mass subunit carboxyl-terminal tail domains is essential for the radial growth and cytoskeletal architecture of axons but not for regulating neurofilament transport rate. J. Cell Biol 163, 1021-1031 (2003).

17. Brown, R.H., Jr. \& Al-Chalabi, A. Amyotrophic Lateral Sclerosis. N. Engl. J. Med 377, 1602 (2017).

18. Rosengren, L.E., Karlsson, J.E., Karlsson, J.O., Persson, L.I. \& Wikkelso, C. Patients with amyotrophic lateral sclerosis and other neurodegenerative diseases have increased levels of neurofilament protein in CSF. J. Neurochem 67, 2013-2018 (1996).

19. Norgren, N., Karlsson, J.E., Rosengren, L. \& Stigbrand, T. Monoclonal antibodies selective for low molecular weight neurofilaments. Hybrid. Hybridomics 21, 53-59 (2002).

20. Norgren, N., Rosengren, L. \& Stigbrand, T. Elevated neurofilament levels in neurological diseases. Brain Res 987, 25-31 (2003).

21. Petzold, A. et al. In vivo monitoring of neuronal loss in traumatic brain injury: a microdialysis study. Brain 134, 464-483 (2011).

22. Zucchi et al. Journal of Neurochemistry.

23. Petzold, A., Keir, G., Warren, J., Fox, N. \& Rossor, M.N. A systematic review and metaanalysis of CSF neurofilament protein levels as biomarkers in dementia. Neurodegener. Dis 4, 185-194 (2007).

24. Petzold, A. et al. Neurofilament ELISA validation. J. Immunol. Methods 352, 23-31 (2010).

25. Kuhle, J. et al. A highly sensitive electrochemiluminescence immunoassay for the neurofilament heavy chain protein. J. Neuroimmunol 220, 114-119 (2010).

26. Kuhle, J. et al. Neurofilament heavy chain in CSF correlates with relapses and disability in multiple sclerosis. Neurology 76, 1206-1213 (2011).

27. Kuhle, J. et al. Serum neurofilament is associated with progression of brain atrophy and disability in early MS. Neurology 88, 826-831 (2017). 
28. Rissin, D.M. et al. Single-molecule enzyme-linked immunosorbent assay detects serum proteins at subfemtomolar concentrations. Nat. Biotechnol 28, 595-599 (2010).

29. Kuhle, J. et al. Serum neurofilament light chain in early relapsing remitting MS is increased and correlates with CSF levels and with MRI measures of disease severity. Mult Scler 22, 1550-1559 (2016).

30. Piehl, F. et al. Plasma neurofilament light chain levels in patients with MS switching from injectable therapies to fingolimod. Mult. Scler, 1352458517715132 (2017).

31. Bacioglu, M. et al. Neurofilament Light Chain in Blood and CSF as Marker of Disease Progression in Mouse Models and in Neurodegenerative Diseases. Neuron 91, 56-66 (2016).

32. Wilke, C. et al. Neurofilament light chain in FTD is elevated not only in cerebrospinal fluid, but also in serum. J. Neurol. Neurosurg. Psychiatry 87, 1270-1272 (2016).

33. Weydt, P. et al. Neurofilament levels as biomarkers in asymptomatic and symptomatic familial amyotrophic lateral sclerosis. Ann. Neurol 79, 152-158 (2016).

34. Lu, C.H. et al. Neurofilament light chain: A prognostic biomarker in amyotrophic lateral sclerosis. Neurology 84, 2247-57 (2015).

35. Meeter, L.H. et al. Neurofilament light chain: a biomarker for genetic frontotemporal dementia. Ann. Clin. Transl. Neurol 3, 623-636 (2016).

36. Novakova, L. et al. Monitoring disease activity in multiple sclerosis using serum neurofilament light protein. Neurology (2017).

37. Martinez-Morillo, E. et al. Neurofilament medium polypeptide (NFM) protein concentration is increased in CSF and serum samples from patients with brain injury. Clin. Chem. Lab Med 53, 1575-1584 (2015).

38. Yilmaz, A. et al. Neurofilament light chain protein as a marker of neuronal injury: review of its use in HIV-1 infection and reference values for HIV-negative controls. Expert. Rev. Mol. Diagn 17, 761-770 (2017).

39. Reiber, H. Flow rate of cerebrospinal fluid (CSF)--a concept common to normal blood-CSF barrier function and to dysfunction in neurological diseases [see comments]. J. Neurol. Sci 122, 189-203 (1994).

40. Idland, A.V. et al. CSF neurofilament light levels predict hippocampal atrophy in cognitively healthy older adults. Neurobiol. Aging 49, 138-144 (2017).

41. Szaro, B.G. \& Strong, M.J. Post-transcriptional control of neurofilaments: New roles in development, regeneration and neurodegenerative disease. Trends Neurosci 33, 27-37 (2010).

42. Mattsson, N., Andreasson, U., Zetterberg, H., Blennow, K. \& Alzheimer's Disease Neuroimaging, I. Association of Plasma Neurofilament Light With Neurodegeneration in Patients With Alzheimer Disease. JAMA Neurol 74, 557-566 (2017).

43. Bischof, A. et al. Serum neurofilament light chain: a biomarker of neuronal injury in vasculitic neuropathy. Ann. Rheum. Dis (2017).

44. Sandelius $\AA$, Zetterberg H, Blennow K, Adiutori R, Malaspina A, Laura M, Reilly MM, Rossor AM. Plasma neurofilament light chain concentration in the inherited peripheral neuropathies. Neurology, (In press).

45. Brownlee, W.J., Hardy, T.A., Fazekas, F. \& Miller, D.H. Diagnosis of multiple sclerosis: progress and challenges. Lancet (London, England) 389, 1336-1346 (2017).

46. Lucchinetti, C.F. et al. Inflammatory cortical demyelination in early multiple sclerosis. $N$. Engl. J. Med 365, 2188-2197 (2011).

47. Huizinga, R., Gerritsen, W., Heijmans, N. \& Amor, S. Axonal loss and gray matter pathology as a direct result of autoimmunity to neurofilaments. Neurobiol. Dis 32, 461-470 (2008). 
48. Trapp, B.D. et al. Axonal transection in the lesions of multiple sclerosis. N. Engl. J. Med 338, 278-285 (1998).

49. Kuhlmann, T., Lingfeld, G., Bitsch, A., Schuchardt, J. \& Bruck, W. Acute axonal damage in multiple sclerosis is most extensive in early disease stages and decreases over time. Brain 125, 2202-2212 (2002).

50. Tallantyre, E.C. et al. Clinico-pathological evidence that axonal loss underlies disability in progressive multiple sclerosis. Mult. Scler 16, 406-411 (2010).

51. Rocca, M.A. et al. Brain MRI atrophy quantification in MS: From methods to clinical application. Neurology 88, 403-413 (2017).

52. Lycke, J.N., Karlsson, J.E., Andersen, O. \& Rosengren, L.E. Neurofilament protein in cerebrospinal fluid: a potential marker of activity in multiple sclerosis. J. Neurol. Neurosurg. Psychiatry 64, 402-404 (1998).

53. Norgren, N. et al. Neurofilament and glial fibrillary acidic protein in multiple sclerosis. Neurology 63, 1586-1590 (2004).

54. Teunissen, C.E. et al. Combination of CSF N-acetylaspartate and neurofilaments in multiple sclerosis. Neurology 72, 1322-1329 (2009).

55. Khalil, M. et al. CSF neurofilament and $\mathrm{N}$-acetylaspartate related brain changes in clinically isolated syndrome. Mult. Scler 19, 436-442 (2013).

56. Arrambide, G. et al. Neurofilament light chain level is a weak risk factor for the development of MS. Neurology 87, 1076-1084 (2016).

57. Petzold, A. et al. CSF neurofilament levels: a potential prognostic marker in Guillain-Barre syndrome. Neurology 67, 1071-1073 (2006).

58. Petzold, A., Steenwijk, M.D., Eikelenboom, J.M., Wattjes, M.P. \& Uitdehaag, B.M. Elevated CSF neurofilament proteins predict brain atrophy: A 15-year follow-up study. Mult. Scler 22, 1154-1162 (2016).

59. Gunnarsson, M. et al. Axonal damage in relapsing multiple sclerosis is markedly reduced by natalizumab. Ann. Neurol 69, 83-89 (2011).

60. Kuhle, J. et al. Fingolimod and CSF neurofilament light chain levels in relapsing-remitting multiple sclerosis. Neurology 84, 1639-1643 (2015).

61. Novakova, L. et al. Cerebrospinal fluid biomarkers of inflammation and degeneration as measures of fingolimod efficacy in multiple sclerosis. Mult Scler 23, 62-71 (2017).

62. Novakova, L. et al. Cerebrospinal fluid biomarkers as a measure of disease activity and treatment efficacy in relapsing-remitting multiple sclerosis. J. Neurochem 141, 296-304 (2017).

63. Axelsson, M. et al. Immunosuppressive therapy reduces axonal damage in progressive multiple sclerosis. Mult. Scler 20, 43-50 (2014).

64. Romme Christensen, J. et al. Natalizumab in progressive MS: results of an open-label, phase 2A, proof-of-concept trial. Neurology 82, 1499-507 (2014).

65. Malmestrom, C., Haghighi, S., Rosengren, L., Andersen, O. \& Lycke, J. Neurofilament light protein and glial fibrillary acidic protein as biological markers in MS. Neurology 61, 17201725 (2003).

66. Rosengren, L.E., Karlsson, J.E., Sjogren, M., Blennow, K. \& Wallin, A. Neurofilament protein levels in CSF are increased in dementia. Neurology 52, 1090-1093 (1999).

67. Sjogren, M. et al. Cytoskeleton proteins in CSF distinguish frontotemporal dementia from AD. Neurology 54, 1960-1964 (2000).

68. Olsson, B. et al. CSF and blood biomarkers for the diagnosis of Alzheimer's disease: a systematic review and meta-analysis. Lancet Neurol 15, 673-684 (2016).

69. Mattsson, N. et al. Cerebrospinal fluid tau, neurogranin, and neurofilament light in Alzheimer's disease. EMBO Mol. Med 8, 1184-1196 (2016). 
70. Zetterberg, H. Neurofilament Light: A Dynamic Cross-Disease Fluid Biomarker for Neurodegeneration. Neuron 91, 1-3 (2016).

71. Zetterberg, $\mathrm{H}$. et al. Association of Cerebrospinal Fluid Neurofilament Light Concentration With Alzheimer Disease Progression. JAMA Neurol, 1-8 (2015).

72. Skillback, T. et al. CSF neurofilament light differs in neurodegenerative diseases and predicts severity and survival. Neurology 83, 1945-1953 (2014).

73. Pijnenburg, Y.A., Verwey, N.A., van der Flier, W.M., Scheltens, P. \& Teunissen, C.E. Discriminative and prognostic potential of cerebrospinal fluid phosphoTau/tau ratio and neurofilaments for frontotemporal dementia subtypes. Alzheimers. Dement. (Amst) 1, 505512 (2015).

74. Andreasson, U., Blennow, K. \& Zetterberg, H. Update on ultrasensitive technologies to facilitate research on blood biomarkers for central nervous system disorders. Alzheimers. Dement. (Amst) 3, 98-102 (2016).

75. Rohrer, J.D. et al. Serum neurofilament light chain protein is a measure of disease intensity in frontotemporal dementia. Neurology 87, 1329-1336 (2016).

76. Weston, P.S.J. et al. Serum neurofilament light in familial Alzheimer disease: A marker of early neurodegeneration. Neurology 89, 2167-2175 (2017).

77. Hansson, O. et al. Blood-based NfL: A biomarker for differential diagnosis of parkinsonian disorder. Neurology 88, 930-937 (2017).

78. Steinacker, P. et al. Neurofilaments in blood and CSF for diagnosis and prediction of onset in Creutzfeldt-Jakob disease. Sci. Rep 6, 38737 (2016).

79. Steinacker, P. et al. Neurofilament as a blood marker for diagnosis and monitoring of primary progressive aphasias. Neurology 88, 961-969 (2017).

80. Nylen, K. et al. CSF -neurofilament correlates with outcome after aneurysmal subarachnoid hemorrhage. Neurosci. Lett 404, 132-136 (2006).

81. Lewis, S.B., Wolper, R.A., Miralia, L., Yang, C. \& Shaw, G. Detection of phosphorylated NF-H in the cerebrospinal fluid and blood of aneurysmal subarachnoid hemorrhage patients. $J$. Cereb. Blood Flow Metab 28, 1261-1271 (2008).

82. Zanier, E.R. et al. Neurofilament light chain levels in ventricular cerebrospinal fluid after acute aneurysmal subarachnoid haemorrhage. J. Neurol. Neurosurg. Psychiatry 82, 157-159 (2011).

83. Traenka, C. et al. Serum Neurofilament Light Chain Levels Are Associated with Clinical Characteristics and Outcome in Patients with Cervical Artery Dissection. Cerebrovasc. Dis 40, 222-227 (2015).

84. Gattringer, T. et al. Serum neurofilament light is sensitive to active cerebral small vessel disease. Neurology 89, 2108-2114 (2017).

85. Bergman, J. et al. Neurofilament light in CSF and serum is a sensitive marker for axonal white matter injury in MS. Neurol. Neuroimmunol. Neuroinflamm 3, e271 (2016).

86. Blennow, K. et al. Traumatic brain injuries. Nat. Rev. Dis. Primers 2, 16084 (2016).

87. McKee, A.C. et al. The first NINDS/NIBIB consensus meeting to define neuropathological criteria for the diagnosis of chronic traumatic encephalopathy. Acta Neuropathol 131, 7586 (2016).

88. Dekosky, S.T., Blennow, K., Ikonomovic, M.D. \& Gandy, S. Acute and chronic traumatic encephalopathies: pathogenesis and biomarkers. Nat. Rev. Neurol 9, 192-200 (2013).

89. Zetterberg, H. et al. Neurochemical aftermath of amateur boxing. Arch. Neurol 63, 12771280 (2006).

90. Neselius, S. et al. CSF-biomarkers in Olympic boxing: diagnosis and effects of repetitive head trauma. PLoS. One 7, e33606 (2012). 
91. Shahim, P. et al. Serum neurofilament light protein predicts clinical outcome in traumatic brain injury. Sci. Rep 6, 36791 (2016).

92. Al Nimer, F. et al. Comparative Assessment of the Prognostic Value of Biomarkers in Traumatic Brain Injury Reveals an Independent Role for Serum Levels of Neurofilament Light. PLoS. One 10, e0132177 (2015).

93. Shahim, P., Zetterberg, H., Tegner, Y. \& Blennow, K. Serum neurofilament light as a biomarker for mild traumatic brain injury in contact sports. Neurology 88, 1788-1794 (2017).

94. Oliver, J.M. et al. Serum Neurofilament Light in American Football Athletes over the Course of a Season. J. Neurotrauma 33, 1784-1789 (2016).

95. Al-Chalabi, A. et al. Amyotrophic lateral sclerosis: moving towards a new classification system. Lancet Neurol 15, 1182-1194 (2016).

96. Brettschneider, J., Petzold, A., Sussmuth, S.D., Ludolph, A.C. \& Tumani, H. Axonal damage markers in cerebrospinal fluid are increased in ALS. Neurology 66, 852-856 (2006).

97. Zetterberg, H., Jacobsson, J., Rosengren, L., Blennow, K. \& Andersen, P.M. Cerebrospinal fluid neurofilament light levels in amyotrophic lateral sclerosis: impact of SOD1 genotype. Eur. J. Neurol 14, 1329-1333 (2007).

98. Mendonca, D.M. et al. Neurofilament heavy subunit in cerebrospinal fluid: a biomarker of amyotrophic lateral sclerosis? Amyotroph. Lateral. Scler 12, 144-147 (2011).

99. McCombe, P.A. et al. Serial measurements of phosphorylated neurofilament-heavy in the serum of subjects with amyotrophic lateral sclerosis. J. Neurol. Sci 353, 122-129 (2015).

100. Li, S. et al. Phosphorylated neurofilament heavy chain levels in paired plasma and CSF of amyotrophic lateral sclerosis. J. Neurol. Sci 367, 269-274 (2016).

101. Steinacker, P. et al. Neurofilaments in the diagnosis of motoneuron diseases: a prospective study on 455 patients. J. Neurol. Neurosurg. Psychiatry 87, 12-20 (2016).

102. De Schaepdryver M. et al. Comparison of elevated phosphorylated neurofilament heavy chains in serum and cerebrospinal fluid of patients with amyotrophic lateral sclerosis. J. Neurol. Neurosurg. Psychiatry (2017).

103. Poesen, K. et al. Neurofilament markers for ALS correlate with extent of upper and lower motor neuron disease. Neurology 88, 2302-2309 (2017).

104. Oeckl, P.P. et al. Multicenter validation of CSF neurofilaments as diagnostic biomarkers for ALS. Amyotroph. Lateral. Scler. Frontotemporal. Degener 17, 404-413 (2016).

105. Gendron, T.F. et al. Phosphorylated neurofilament heavy chain: A biomarker of survival for C9ORF72-associated amyotrophic lateral sclerosis. Ann. Neurol 82, 139-146 (2017).

106. Lehmer, C. et al. Poly-GP in cerebrospinal fluid links C9orf72-associated dipeptide repeat expression to the asymptomatic phase of ALS/FTD. EMBO Mol. Med 9, 859-868 (2017).

107. Feneberg, E., P. Oeckl, P. Steinacker, F. Verde, C. Barro, P. Van Damme, E. Gray, J. Grosskreutz, C. Jardel, J. Kuhle, S. Koerner, F. Lamari, M. del Mar Amador, B. Mayer, C. Morelli, P. Muckova, S. Petri, K. Poesen, J. Raaphorst, F. Salachas, V. Silani, B. Stubendorff, M. Turner, M. Verbeek, J. Weishaupt, P. Weydt, A. C. Ludolph and M. Otto (2017). "Multicenter evaluation of neurofilaments in early symptom onset amyotrophic lateral sclerosis." Neurology, (In press).

108. Boylan, K.B. et al. Phosphorylated neurofilament heavy subunit (pNF-H) in peripheral blood and CSF as a potential prognostic biomarker in amyotrophic lateral sclerosis. J. Neurol. Neurosurg. Psychiatry 84, 467-472 (2013).

109. Menke, R.A. et al. CSF neurofilament light chain reflects corticospinal tract degeneration in ALS. Ann. Clin. Transl. Neurol 2, 748-755 (2015).

110. Volkening, K., Leystra-Lantz, C., Yang, W., Jaffee, H. \& Strong, M.J. Tar DNA binding protein of 43 kDa (TDP-43), 14-3-3 proteins and copper/zinc superoxide dismutase (SOD1) interact 
to modulate NFL mRNA stability. Implications for altered RNA processing in amyotrophic lateral sclerosis (ALS). Brain Res 1305, 168-182 (2009).

111. Steinacker, P. et al. Diagnostic and prognostic significance of neurofilament light chain NFL, but not progranulin and S100B, in the course of amyotrophic lateral sclerosis: Data from the German MND-net. Amyotroph. Lateral. Scler. Frontotemporal. Degener 18, 112-119 (2017).

112. Halbgebauer, S., Ockl, P., Wirth, K., Steinacker, P. \& Otto, M. Protein biomarkers in Parkinson's disease: Focus on cerebrospinal fluid markers and synaptic proteins. Mov Disord 31, 848-860 (2016).

113. Eusebi, P. et al. Diagnostic utility of cerebrospinal fluid alpha-synuclein in Parkinson's disease: A systematic review and meta-analysis. Mov Disord 32, 1389-1400 (2017).

114. Holmberg, B., Rosengren, L., Karlsson, J.E. \& Johnels, B. Increased cerebrospinal fluid levels of neurofilament protein in progressive supranuclear palsy and multiple-system atrophy compared with Parkinson's disease. Mov Disord 13, 70-77 (1998).

115. Brettschneider, J. et al. Neurofilament heavy-chain NfH(SMI35) in cerebrospinal fluid supports the differential diagnosis of Parkinsonian syndromes. Mov Disord 21, 2224-2227 (2006).

116. Hall, S. et al. Accuracy of a panel of 5 cerebrospinal fluid biomarkers in the differential diagnosis of patients with dementia and/or parkinsonian disorders. Arch. Neurol 69, 14451452 (2012).

117. Magdalinou, N.K. et al. A panel of nine cerebrospinal fluid biomarkers may identify patients with atypical parkinsonian syndromes. J. Neurol. Neurosurg. Psychiatry 86, 1240-1247 (2015).

118. Rojas, J.C. et al. Plasma neurofilament light chain predicts progression in progressive supranuclear palsy. Ann. Clin. Transl. Neurol 3, 216-225 (2016).

119. Byrne, L.M. et al. Neurofilament light protein in blood as a potential biomarker of neurodegeneration in Huntington's disease: a retrospective cohort analysis. Lancet Neurol 16, 601-609 (2017).

120. Wild, E.J., Petzold, A., Keir, G. \& Tabrizi, S.J. Plasma neurofilament heavy chain levels in Huntington's disease. Neurosci. Lett 417, 231-233 (2007).

121. Constantinescu, R., Romer, M., Oakes, D., Rosengren, L. \& Kieburtz, K. Levels of the light subunit of neurofilament triplet protein in cerebrospinal fluid in Huntington's disease. Parkinsonism. Relat Disord 15, 245-248 (2009).

122. Niemela, V., Landtblom, A.M., Blennow, K. \& Sundblom, J. Tau or neurofilament lightWhich is the more suitable biomarker for Huntington's disease? PLoS. One 12, e0172762 (2017).

123. Cousins, D.A. \& Grunze, H. Interpreting magnetic resonance imaging findings in bipolar disorder. CNS. Neurosci. Ther 18, 201-207 (2012).

124. Jakobsson, J. et al. Elevated concentrations of neurofilament light chain in the cerebrospinal fluid of bipolar disorder patients. Neuropsychopharmacology 39, 2349-2356 (2014).

125. Isgren, A. et al. Markers of neuroinflammation and neuronal injury in bipolar disorder: Relation to prospective clinical outcomes. Brain Behav. Immun 65, 195-201 (2017).

Figure 1 | Structure, assembly and release of neurofilaments. [Au: The figure needs an overall title. Suggestion OK?] a | Domain structure and post-translational modifications of neurofilament subunits ${ }^{11}$. Neurofilament light chain (NfL), neurofilament medium chain (NfM), neurofilament 
heavy chain $(\mathrm{NfH}), \alpha$-internexin and peripherin are the subunits of neurofilaments in the mature nervous system. All neurofilament subunits include a conserved $\alpha$-helical rod domain that comprises several coiled-coils, and variable amino-terminal globular head regions and carboxyterminal tail domains. $\mathrm{NfM}$ and $\mathrm{NfH}$ subunits are unique among the intermediate filament proteins in that they have long carboxy-terminal domains with multiple lys-ser-pro repeats that are heavily phosphorylated. Phosphorylation and $O$-linked glycosylation sites on neurofilament subunits are shown. E segment, glutamic-acid-rich segment; E1, glutamic-acid rich segment 1; E2, glutamicacid-rich segment 2. [Au: We will use the three-letter amino acid codes in the figure, so the oneletter codes do not need to be in the legend.] b | Neurofilament assembly. Neurofilament protein monomers form parallel coiled-coil heterodimers ${ }^{11}$. Two dimers form staggered antiparallel tetramers through interactions between coil domains $1 a, 1 b$ and $2 a^{12}$. The lateral association of eight tetramers results in formation of cylindrical structures known as unit-length filaments that have a diameter of $\sim 16 \mathrm{~nm}$ and a length of $\sim 60 \mathrm{~nm}$. Gradual end-to-end annealing of these unitlength filaments results in filament elongation, which is followed by radial compaction to form the mature, long neurofilament polymer with a diameter of $\sim 10 \mathrm{~nm}$. Tail domains of $\mathrm{NfM}$ and $\mathrm{NfH}$ radiate outwards from the filament core because of the extensive negative charges from large numbers of glutamic acid and phosphorylated serine and threonine residues.

[Au: I suggest that part $\mathrm{c}$ is a separate figure, because it deals with a slightly different aspect to the structure, and this avoids adding to what is already a very long figure legend.]

Figure 2 | Neurofilament release after axonal damage. When an axon is damaged, cytoskeletal proteins, including neurofilaments, are released into the extracellular space and subsequently into the CSF and, at lower concentrations, into the blood. First-generation (immunoblots) and secondgeneration (ELISA) immunoassays can typically detect neurofilament in the CSF. Third-generation (electrochemiluminescence) and, in particular, fourth-generation (Single molecue array) immunoassays can reliably measure blood levels of neurofilament light which was not possible from the blood with ELISA.

[Au: I think the information in your figure 2 would be better presented in a text box because it is really just text. I have suggested a format for this below.]

\section{Box 1 | Relevance of neurofilaments to neurological disorders}


Neurofilaments have been studied in several neurological disorders, and in many, good evidence indicates their diagnostic and prognostic value and/or their use for monitoring treatment responses. The disorders reviewed here are:

- Multiple sclerosis

- Dementia

- $\quad$ Stroke

- Traumatic brain injury

- Amyotrophic lateral sclerosis

- Parkinson disease

- Huntington disease

- Bipolar disorder (limited evidence for clinical utility).

In addition, neurofilaments could be of relevance in many other neurological disorders, but their association with these disorders has not been studied. Such disorders include:

- Epilepsy

- Encephalitis

- Meningitis

- Hypoxic brain injury

- Optic neuropathies

- Intracranial pressure

- Neurotoxicity

- Peripheral neuropathies including Guillain-Barré Syndrome, CIDP and CMT. 